\title{
Self-Powered Passive Adaptive Control of Pitch Angle and Betz-Shaped Wind Tunnel
}

\author{
S. C. $\mathrm{Li}$ \\ School of Engineering \\ Warwick University \\ Coventry CV4 7AL, UK \\ E-mail: S.Li@warwick.ac.uk
}

\begin{abstract}
Owing to both the high cost and the complexity, for small turbines, the control of pitch angle is usually not a option though it could keep turbine working in high efficiency zone while wind speed changes.

In order to solve this problem, a self-powered passive adaptive control system (SPACS) for pitch angle adjustment has been developed. Together with this SPACS idea, a Betz-shaped wind tunnel has also been developed which can reduce the size and running cost significantly if compared with conventional wind tunnels. In this paper the main technical principles will be presented together with some conceptual sketches and initial results.
\end{abstract}

\section{Key words}

Small wind turbine, self-powered passive adaptive control system, pitch angle control, Betz-shaped wind tunnel

\section{Introduction}

It is possible to increase the use of small turbines remarkably if its bottle neck problems can be solved. One of such problems is the high capital cost associated with pitch-angle control systems.

In order to develop a robust system, which should be low cost, low maintenance and high performance, an innovative concept has been created by the author in early 2000's [1]. It was then offered as research projects for undergraduate and postgraduate projects at Warwick University in the last 5 years.

The author would like to present this concept idea here with a hope that this new idea together with the Betzshaped wind tunnel can help solving the problems mentioned above, promoting small wind turbines

\section{Main Principles of the System}

The principle of the SPACS is as follows. The aerodynamic force acting upon on the blade is used as control power for adjusting the pitch angle against the variation of wind speed while the balance force is provided by a set of specially designed springs. The characteristics of the springs are such determined that makes the blade always maintain at the desired (high efficient) pitch angles while wind speed changes. That is, the operating points of the turbine are always on the ridge of the performance surface as shown schematically by Fig. 1.

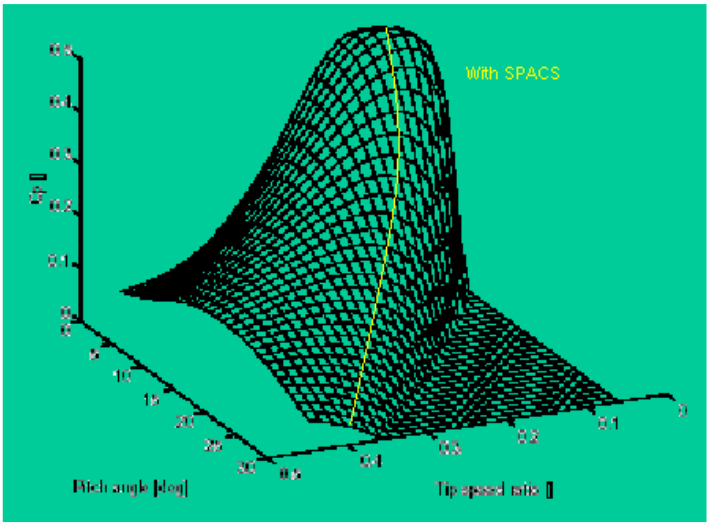

Fig.1 SPACS makes the turbine always operating at high performance zone

Its transient process must be smooth and stable; and the system must also be robust, simple, low cost and low maintenance in order to beat the high cost associated with conventional pitch angle control system.

\section{Technical Issues}

In order to realise those functions mentioned above, several unique techniques have been developed.

The passive pitch control characteristics of the SPACS should be determined as follows. At first, the spring characteristics are determined by using a static analysis. Then the damper characteristics are determined from the vibration behaviour of the system for both static and turbulent winds. Finally, a stability analysis follows in order to specify the limits of the physical parameters of the control system for stability. During the above processes, the interplay of three centres of blades (i. e. the aerodynamic force centre, the rotating centre and the mass centre) plays an essential role.

This passive control system only consists of spring and damper. Once the turbine blade is designed, the determination of the characteristics of these two elements 
is the primary concern for the system's energy performance and dynamic stability etc.

\section{A. Three Centres}

There are several stages for the determination of the final characteristics of the control system. The first stage is the determination of the spring characteristics which involves the determinations of

1) the centre of mass of the blade;

2) the centre of blade rotation;

3) the centre of aerodynamic force.

Once the spring characteristics are determined, the next stage is the analysis of the vibration features of the system through which we can determine the damper characteristics. Finally, the limits of stability of the system are determined by using the Routh-Hurwitz stability criterion.

The determination of the mass centre is important as it affects the dynamic performance of the SPACS. If the rotation centre coincides with the mass centre, the system will have the maximum response speed.

Usually the blade is designed with the blade axis at the $25 \%$ of the chord which is roughly the aerodynamic centre. This will minimise the control force for pitch angle adjustment. Therefore, it is hoped that the mass centre could be close to this location. This could be achieved by various means during blade manufacturing process, say by changing the weight distributions in the blades etc. However, the main requirement for a control system is fast response and stability. These two parameters are contradict to each other. A compromise has to be made through the final tuning of these three centres which is a delicate process.

\section{B. Spring Characteristics}

For given wind speeds, the aerodynamic forces acting upon the blades can be determined easily based on the required optimal pitch angles against wind speeds. According to thus determined optimised condition, the characteristics of the spring can be determined.

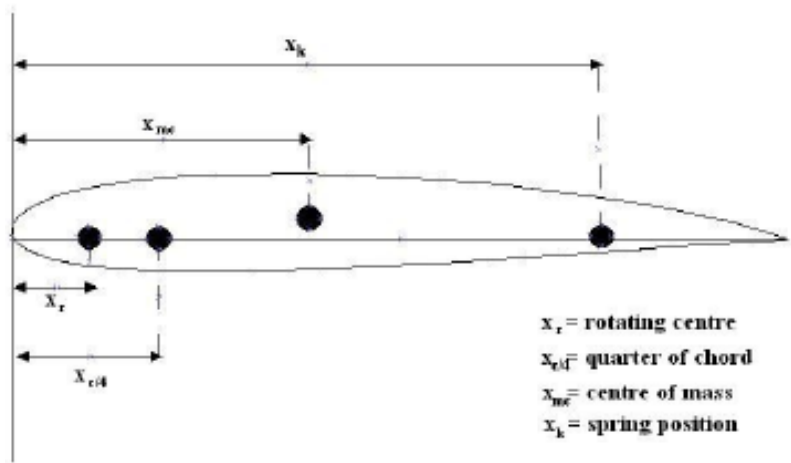

Fig2. Three centres for case 3

Take one of the student projects as an example [2], the determination of the spring characteristics can be performed as follows.

The pitching angles against wind speed has to be calculated in order to determine the necessary spring compression for maintaining the angle of attack at required position while wind speed changes.

Three different cases are considered. In the first case, the blade axis (i.e. the axis of blade pitching) is placed at the mass centre. In the second case, the blade axis is at the quarter of the chord (c/4) where is roughly the aerodynamic centre and in the third case it is at an arbitrary point between the leading edge and the quarter of the chord.

Figure 2 illustrates the positions of the three centres for case 3, where $X r$ is the arbitrary selected pitching point, and $X m c$ is the centre of mass.

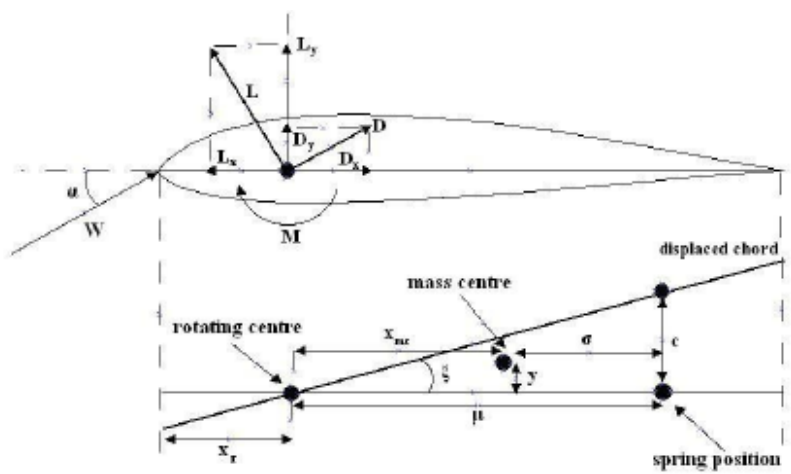

Fig3. Spring calculation (case 2)

Figure 3 Illustrates the arrangement used for the calculations of blade moment and spring characteristics.

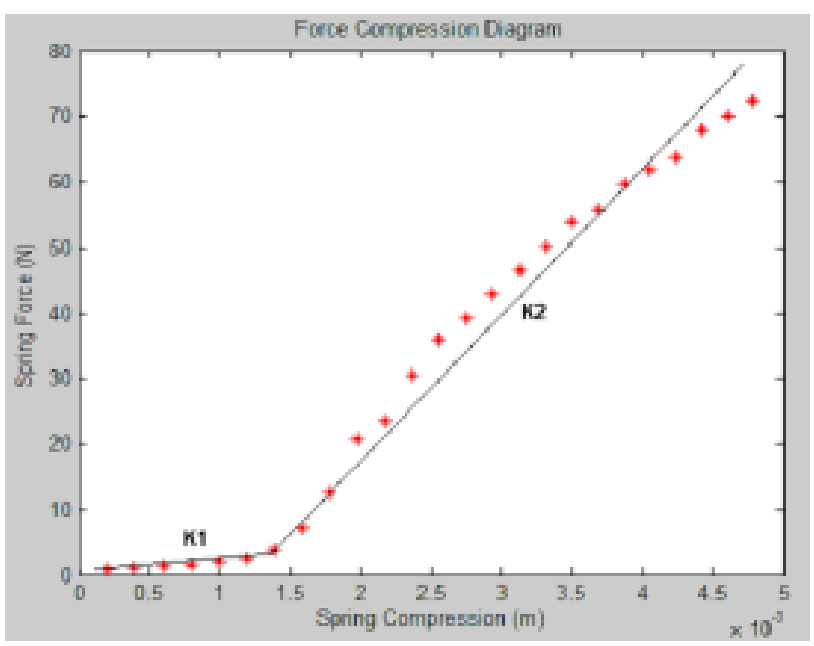

Fig4. Required spring characteristics which can be approximately replaced by two linear springs

The resultant characteristic of the spring is shown by the dots in Fig 4. It can be seen that by carefully choosing the positions of these three centres in particular the pitching centre the spring function can be readily realised by a set of two linear springs.

\section{Dynamic Stability}

Given spring(s) introduced into a dynamic system, it is essential to ensure the system's stability within the operating range.

Forced-vibration has been analysed for the system with two springs. The forced term in the differential equation is expressed by a sinusoidal function of the form

$$
F(t)=M_{0} \cos \omega t
$$

where $\omega$ is the excitation frequency, which is ranged between $0-20 \mathrm{~Hz}$ and $M_{0}$ is the magnitude of the moment caused on the blade due to the turbulence level of the wind. According to the safety standards 61400-2 for small wind turbines (the International 
Electrotechnical Commission, IEC), the turbulence intensity is set to a range of $10-20 \%$ of the design wind speed.

As we can see from the analysis below, the system's dynamic behaviour is subject to which spring being involved. Therefore, the analysis is performed for both springs in order to obtain a compromised solution.

The amplitude and phase responses of the system can be evaluated for different values of the damping coefficient $\zeta$. We define a frequency ratio $\Omega$ as the ratio of the forced frequency $(\omega)$ to the natural frequencies $\left(\omega_{\mathrm{n} 1}\right.$ and $\omega_{\mathrm{n} 2}$ for springs 1 and 2 respectively). If we select the same $\Omega$ value, say $\Omega_{1}=4$ and $\Omega_{2}=4$ for the first and second spring respectively, the system will behave virtually the same. However, owing to the system natural frequencies for both springs being different with $\omega_{\mathrm{n} 1}=56.45 \mathrm{rad} /$ sec and $\omega_{\mathrm{n} 2}=227.5 \mathrm{rad} / \mathrm{sec}$, for the same forced frequency $\omega$ the system may have very different behaviour. For example, let's consider $\omega_{1}$ and $\omega_{2}$ ranging between $0-20 \mathrm{~Hz}$ (i.e. $0-125.6 \mathrm{rad} / \mathrm{s}$ ) which is recommended by the IEC standards. Completely different responses are revealed. For example, Fig.5 shows the different patterns of the amplitude response for underdamping cases; and Fig. 6 shows the different patterns of the phase response for over-damping cases.
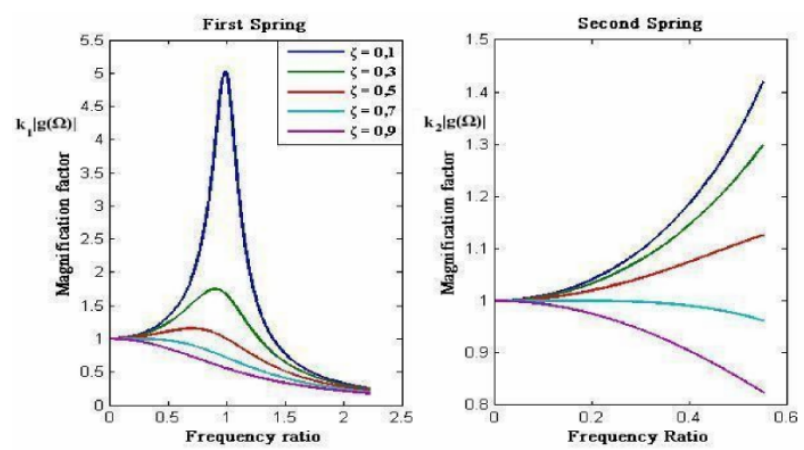

Fig5. Under-damped amplitude response for $\omega$ ranging between $0-20 \mathrm{~Hz}$
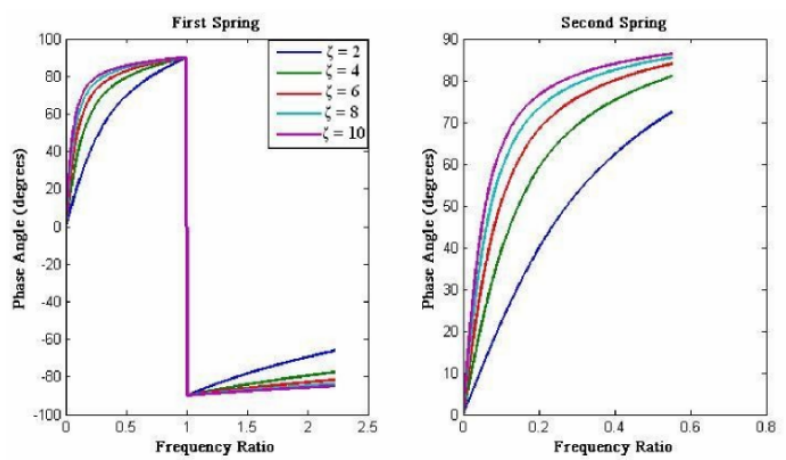

Fig6. Over-damped phase response for $\omega$ ranging between $0-20 \mathrm{~Hz}$

For the first spring, its amplitude response is very high when the excitation frequency coincides with the natural frequency (resonance). Also, the phase angle response is positive until it reaches the resonant frequency. All these features are contrast to those for the second spring. Furthermore, the system will also behave differently when the system operates on the first spring with the frequency ratio changing from less than unity to greater than unity. For the second spring, things are much simpler.

In order to determine a proper value of damping for the system. Further investigations by observing its forced response to sinusoidal excitations have been performed firstly for an average wind frequency of $10 \mathrm{~Hz}$ then for extreme frequencies of $1 \mathrm{~Hz}$ and $20 \mathrm{~Hz}$ respectively. The turbulence intensity is also included in the investigations. Once the damping value is determined for the system a further stability analysis for the full range of operation conditions is performed by using the Routh-Hurwitz approach.

\section{Routh-Hurwitz Approach}

The purpose of this stability analysis is to determine the limits of the parameters of the system within which the system is stable. These parameters involve almost all the physical data of the system such as the moment of inertia, the spring stiffness, the damping coefficient $\zeta$ and the distance at which the spring and damper are mounted. By knowing the limits we can design and optimise the system to its most reliable, cost-effective, energyeffective and stable conditions.

In order to perform this study, the closed loop transfer function of the system needs to be established with one or more feedback paths. The transfer function determines the ratio of the output signal with the input signal. The denominator of the transfer function is called the characteristic equation of the system whose roots determine the stability of the system.

Taking the work by a project student as schematically shown by Fig.5, this is explained briefly as follows.

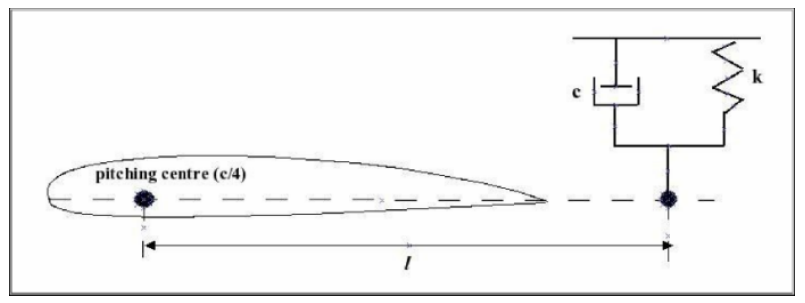

Fig5. One of projects showing the control system using simple damping and spring devices

If we designate $\theta$ as the pitch angle, the general differential equation of the system is

$$
J \ddot{\theta}+c l^{2} \dot{\theta}+k l^{2} \theta=F(t)
$$

Let $\omega$ be the angular velocity of the blade rotating around its axis. We can choose a set of state variables for the system as follows.

$$
\begin{gathered}
\omega=\dot{\theta} \\
\dot{\omega}=\frac{1}{J}\left(-c l^{2} \omega-k l^{2} \theta+F(t)\right)
\end{gathered}
$$

Therefore the general block diagram can be constructed as shown in figure 6 .

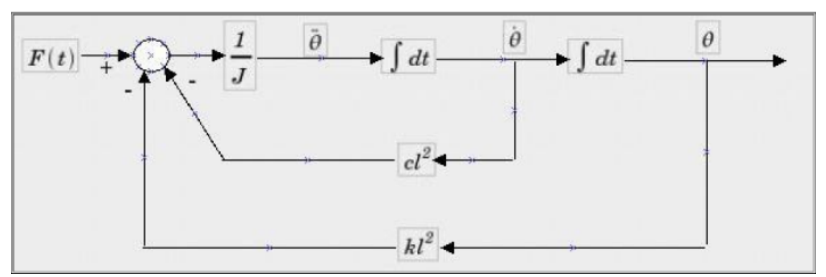

Fig6. Block diagram of a pitch control system 
The transfer function therefore, which is the output over the input will be

$$
\frac{\Theta(s)}{F(s)}=\frac{1}{J s^{2}+c l^{2} s+k l^{2}}
$$

The denominator of the transfer function is referred as the characteristic equation of the system. In this case it is a $2^{\text {nd }}$ order polynomial equation with constant coefficients. The roots of the characteristic equation of a control system determine its stability. For the stability analysis of the passive pitch control system the RouthHurwitz approach has been used which is based on the Hurwitz criterion. The analysis found that

1. Even if the spring and damper are mounted in the leading edge of the aerofoil (i.e. $l<0$, referring to Fig.5), the system will continue to be stable;

2. The passive pitch control system will be unstable only if the mounting point of the spring and the damper is exactly on the pitching centre of the blade. That is, the mounting distance $l$ becomes 0 and therefore, the damping term and the natural frequency term in the differential equation of motion vanish. Physically this means no spring and damper devices.

3. From this analysis it is seen that the system will be very stable even if equipped with simple damper and spring as shown by the schematic diagram in Fig5.

\section{E. Betz-shaped Wind Tunnel ${ }^{l}$}

It is commonly known that in order to satisfy the infinite boundary condition of flow similarity, the diameter of the wind tunnel for testing models or prototypes of wind turbines must be several times larger than the stream-tube (or flow-tube) generated by the turbine flow. For wind turbine, the ideal shape of this flow-tube is the Betzshape (Fig.7) when the turbine can generate the theoretically maximum power (energy) equivalent to an efficiency of $\eta=\frac{16}{27}=59.3 \%$. At this condition, the velocities will have the following relationships:

and

$$
\alpha=\frac{V_{2}}{V_{1}}=\frac{1}{3}
$$

$$
V=\frac{\mathrm{V}_{1}+\mathrm{V}_{2}}{2}
$$

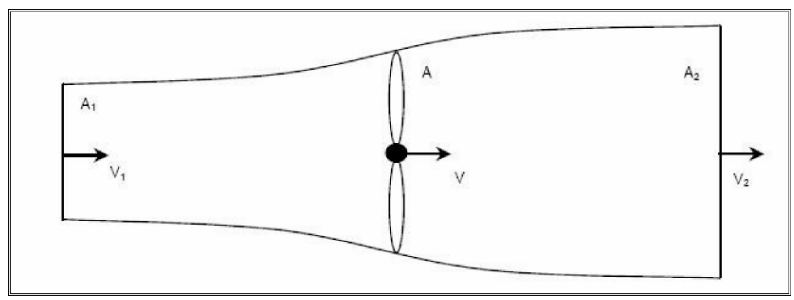

Fig7. Ideal Betz flow through a wind turbine

If the diameter of the turbine being tested is $D$, then the exit diameter $D_{2}$ of the stream-tube will be

$$
D_{2}=\sqrt{2} D
$$

\footnotetext{
${ }^{1}$ This work together with CFD simulation has won the $2^{\text {nd }}$ Prize for 2007 CD-Adapco European Competition
}

For satisfying the infinite boundary condition, the crosssection dimension $D_{t}$ of the tunnel must be, say 5 fold of this size, i.e.

$$
D_{t}=5 \sqrt{2} D
$$

However, owing to the size of the wind turbines being such large even for testing small turbines, satisfaction of this condition will push the size of wind tunnel to extreme conditions. Considering the long wake flow (at least 10 time of the turbine diameter from our CFD results), a decent wind tunnel for wind turbine (even scaled-down models) test is very expensive if not economically feasible.

For the first time, a Betz-shaped tunnel has be proposed by the author [1] and designed and built up as student projects [e.g. 3 and 4] in the Wind Turbine Lab of Warwick University, referring to Figs.8, 9, 10 and 11.

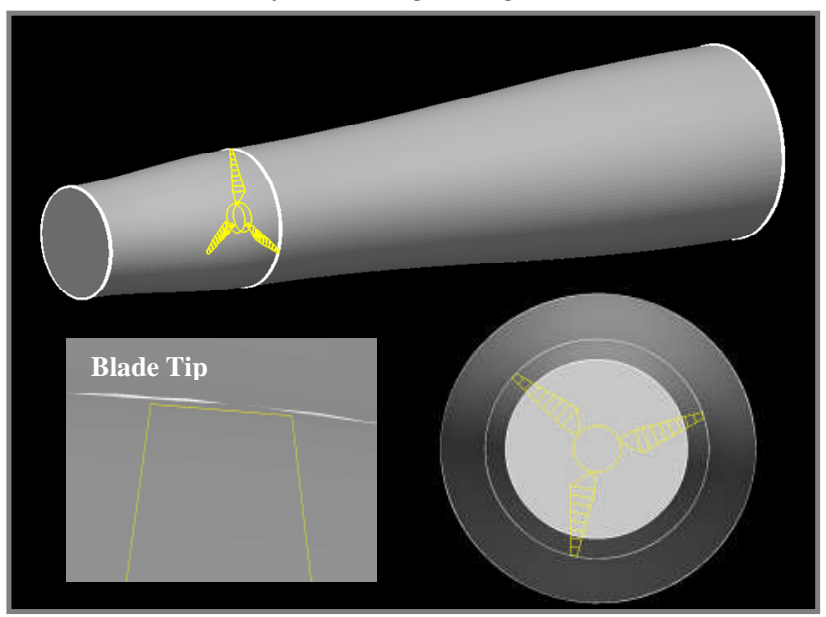

Fig8. Betz-shaped tunnel developed from CFD simulation

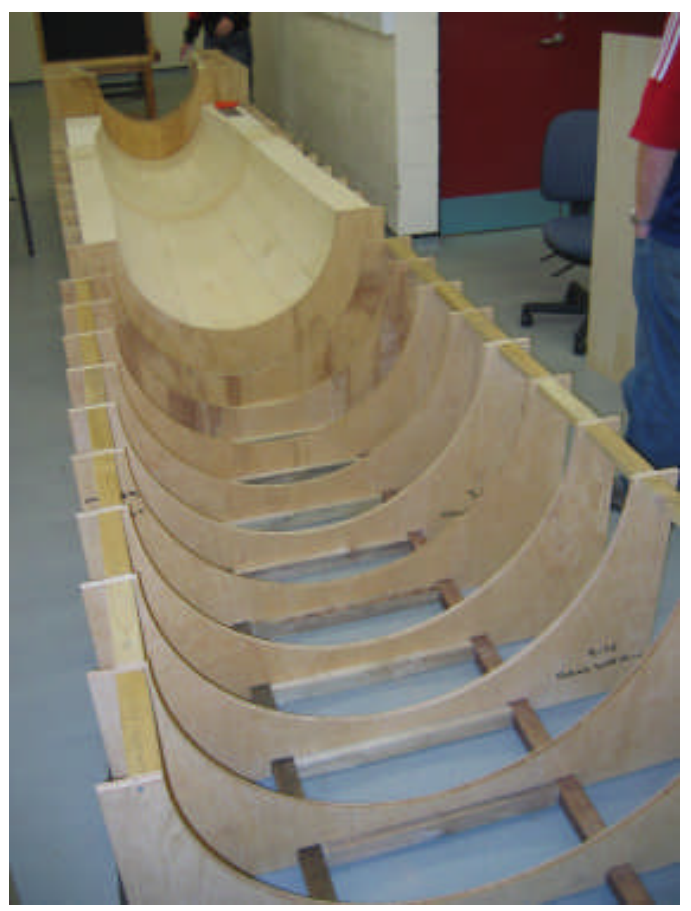

Fig. 9. Manufacturing process of the Betz-shaped wind tunnel at Warwick University 


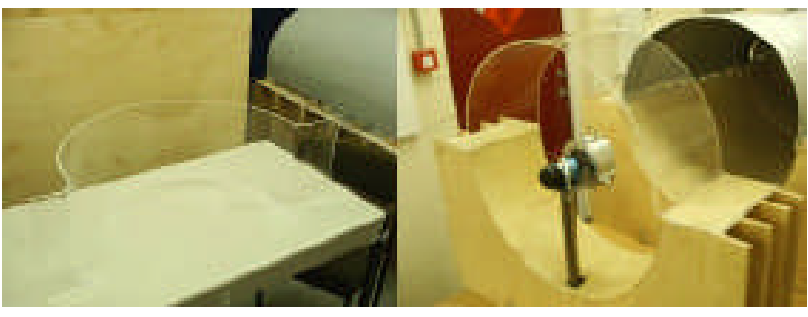

Fig10. Test-section of Betz-shaped tunnel being assembled

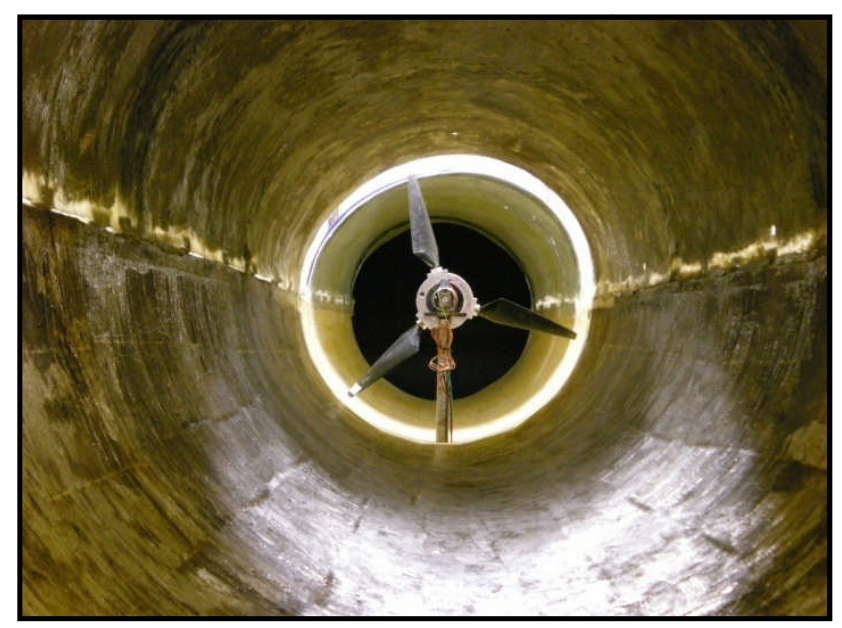

Fig11. A small turbine mounted in the Betz-shaped tunnel

\section{Some Design Examples}

Some designs produced steming from these ideas are briefly introduced here.

\section{A. Three Blade Example}

As a MEng group project, a three blade model equipped with a SPACS system has been designed as shown by Fig12. Its specifications are listed in Table 1.

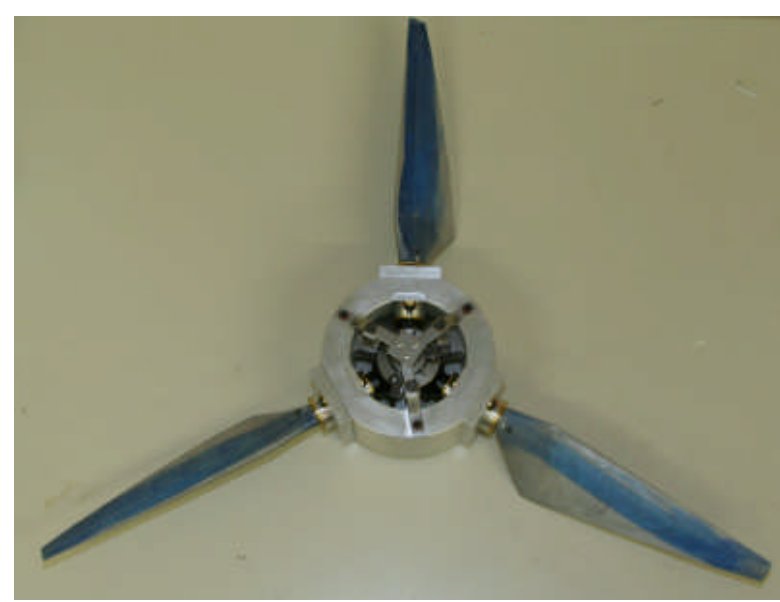

Fig 12. A 3-blade wind-turbine model equipped with a SPACS system in the hub

\section{B. Low-Wind-Speed-Starting Turbine}

For small turbines in particular those installed in urban areas, they must be able to start generating power at low wind-speeds such as $2-3 \mathrm{~m} / \mathrm{sec}$. This is currently a bottleneck problem too as most small turbines have a starting wind-speed around $3-3.5 \mathrm{~m} / \mathrm{sec}$. In response to such a challenge, one of the MEng group project was producing a low-wind-speed turbine equipped with a SPACS system [5].

Table 1 Specifications of 3 Blade Turbine - One of projects -

\begin{tabular}{|l|l|}
\hline Turbine diameter: & $700 \mathrm{~mm}$ \\
\hline Hub diameter: & $150 \mathrm{~mm}$ \\
\hline Blade length: & $235 \mathrm{~mm}$ \\
\hline Number of blades: & 3 \\
\hline Design wind speed : & $7.5 \mathrm{~m} / \mathrm{sec}$ \\
\hline Rotational speed : & $1500 \mathrm{rpm}$ \\
\hline Angular velocity : & $157.1 \mathrm{rad} / \mathrm{sec}$ \\
\hline Tip speed velocity : & $54.98 \mathrm{rad} / \mathrm{sec}$ \\
\hline Tip speed ratio: & 7.33 \\
\hline Design angle of attack : & $7^{\circ}$ \\
\hline Blade root diameter: & $20 \mathrm{~mm}$ \\
\hline Max. blade chord length: & $80 \mathrm{~mm}$ \\
\hline Max. blade thickness: & $12 \mathrm{~mm}$ \\
\hline Min. blade chord length : & $20 \mathrm{~mm}$ \\
\hline Min. blade thickness: & $3 \mathrm{~mm}$ \\
\hline
\end{tabular}

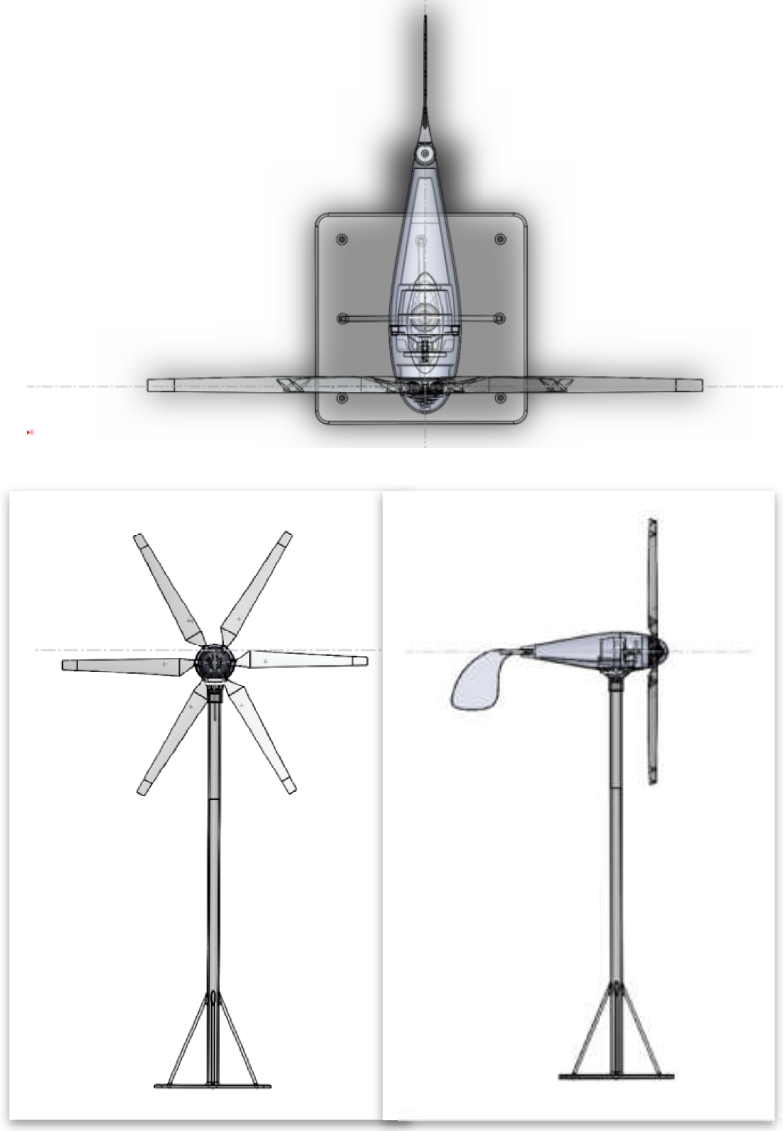

Fig 13. A Low-velocity starting turbine equipped with a SPACS system.

The overall design is shown by Fig13. Figure 14 shows the turbine design. 


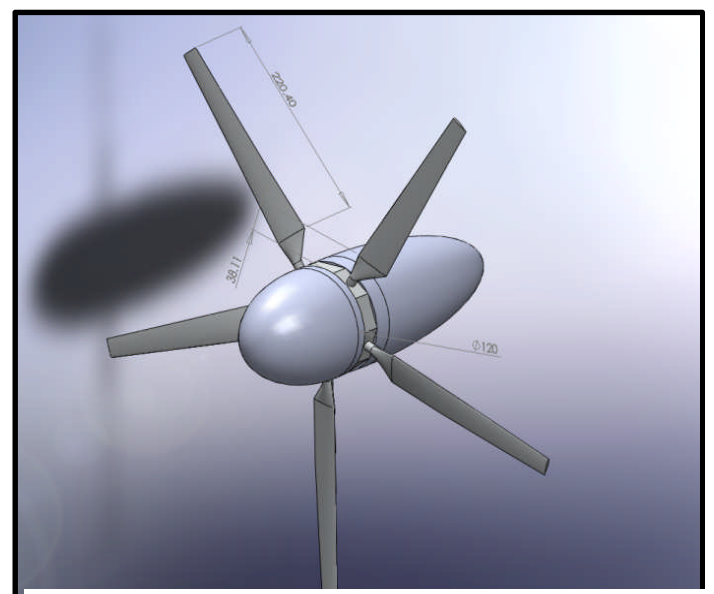

Fig 14. Dimensions of turbine with a SPACS system.

\section{Concluding Remarks}

Based on the author's original idea, several design projects have been conducted at Warwick University. In this paper, a brief concept of the SPACS system together with possible applications to small wind turbines has been introduced briefly. The following concluding remarks can be drawn.

1. SPACS system is a promising approach for tackling the bottle-neck problem of small wind turbines.

2. It offers a robust and high performance system at low-cost.

3. Together with the innovative idea of Betz-shaped wind tunnel, it is hoped that a much more popular use of small wind turbine will be realised by employing such innovative concepts.

4. It is expected to develop this into commercial products with potential industrial partners.

\section{Acknowledgement}

Thanks to all students who have chosen this challenging project as their research projects at Warwick University. They are (in the order of year):

\section{4-2005}

$3^{\text {rd }}$ year project:

I Swan, P Dunstan and A Pearson

MSc project:

K. Papadikis, A. D. Vassatis.

\section{5-2006}

MSc project:

J Gopalan

MEng group project:

P. Dunstan (group leader), E.Borgnis, J.Chapman, A. Chiu, P.Hallgarth, I.Swan, H.Perrin and N.A.Wensley

\section{7-2008}

MEng group project:

P. Lynch (group leader), I. Osman, C. Rison, J. Atkinson, A. Hirani, P. Vernon, C. Charlwood, O. Oshodi

\section{7-2008}

MEng group project:

O. Kuyoro (group leader), S. Dagadu, L. Dixon, B. Pace, S. Saxby, D. Starkey, H. K. Wong, T. F. Yu

\section{References}

[1] S. Li, 'Novel Wind Turbine: Design, Manufacturing and Experimental Study', Student Project SCL.2, $20^{\mathrm{h}}$
August 2004, EUO Student Project Proposals, School of Engineering, University of Warwick, (2004).

[2] K. Papadikis, 'Mathematical Modelling of a Passively Adaptive Pitch Control System', 2004-05 MSc Project, School of Engineering, Warwick University, (2005).

[3] A. D. Vassatis, 'Design and Computational Modelling of a Novel Micro Wind Turbine with the Purpose of Verifying it in a Betz Shaped Wind Tunnel', 2004-05 MSc Project, School of Engineering, Warwick University, (2005).

[4] P. Dunstan (Group leader), 'Development of a Small Scale Novel Wind Turbine with a Self Powered Passive-adaptive Control System', 2005-06 MEng Group Project, School of Engineering, Warwick University, (2005).

[5] O Kuyoro (Group leader), 'Development of a Micro Wind Turbine with Passive Adaptive System‘, 2008-09 MEng Group Project, School of Engineering, Warwick University, (2009). 